\title{
Intentional intravenous mercury injection
}

\author{
G Yudelowitz, MB BCh \\ Tambo Memorial Hospital, Boksburg, South Africa \\ Corresponding author: G Yudelowitz (gregyudels@gmail.com)
}

\begin{abstract}
Mercury toxicity is commonly associated with vapour inhalation or oral ingestion, for which there exist definite treatment options. Intravenous mercury injection is rarely seen, with few documented cases. Treatment strategies are not clearly defined for such cases, although a few options do show benefit. This case report describes a 29-year-old man suffering from bipolar disorder, who presented following self-inflicted intravenous injection of mercury. Clinical and radiographic features, possible adverse clinical sequelae in preexisting mental illness and further complications are discussed, as well as possible treatment strategies in light of relevant literature.
\end{abstract}

S Afr Med J 2017;107(2):112-114. DOI:10.7196/SAMJ.2017.v107i2.12046

Three forms of mercury exist: elemental, inorganic and organic, all of which may be toxic with clinical consequences, depending on the type of exposure. Elemental mercury poisoning usually occurs via vapour inhalation, as mercury is well absorbed through the lungs. The central nervous system is then the major site of deposition. Elemental mercury is the well-known silver liquid and usually causes pulmonary, neurological and nephrological toxicity. ${ }^{[1]}$ Sources of exposure are mainly occupation related and include thermometer and barometer manufacturing, and gold mining, where liquefied elemental mercury may be used to concentrate gold. Other sources are dentistry, as amalgam dental fillings consist of mercury, and metal refineries. ${ }^{[1]}$

Inorganic mercury is present in an oxidised state, allowing the formation of mercury salts and, if ingested, may cause gastroenteritis and renal failure. The kidneys are the final site for deposition. Sources of inorganic mercury exposure may include occupational exposure in the electroplating industry and exposure to laboratory reagents. ${ }^{[1]}$

In this case report, intravenous complications, treatment strategies and possible neuropsychiatric manifestations, where there is preexisting mental disease, are explored in a South African (SA) setting. As far as the author is aware this is the first reported case in SA.

\section{Case report}

A 29-year-old man presented to Tambo Memorial Hospital, Boksburg, SA in June 2016 after having injected mercury intravenously his right and left arms 10 weeks earlier in an attempted suicide. The patient had presented owing to nonspecific symptoms of loss of appetite, occasional diarrhoea and fatigue. On further questioning a history of intravenous mercury injection was uncovered.

The patient was known to suffer from bipolar mood disorder type 1, diagnosed in 2010, with subsequent default of treatment in the same year, soon after initiating treatment. Three previous suicide attempts were reported. At this presentation, the patient was not expressing suicidal ideation or features of major depression.

Clinically the patient was stable, apyrexial and not distressed. Systemic examination was largely unremarkable, except for multiple areas of induration and granuloma formation on the right and left forearms.

Radiographs of the forearms, chest and abdomen were obtained (Figs 1 -4). Images showed numerous metallic hyperdensities in the

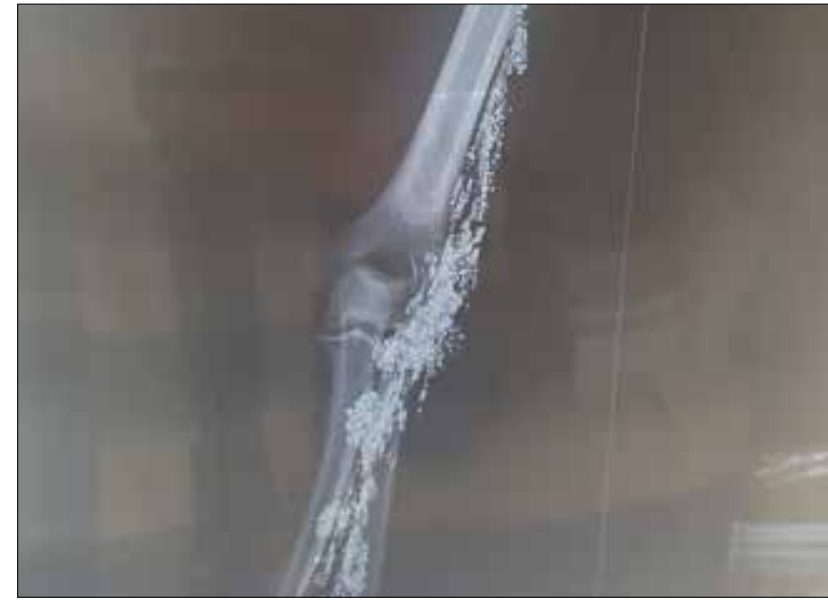

Fig. 1. Radiograph of the right forearm.

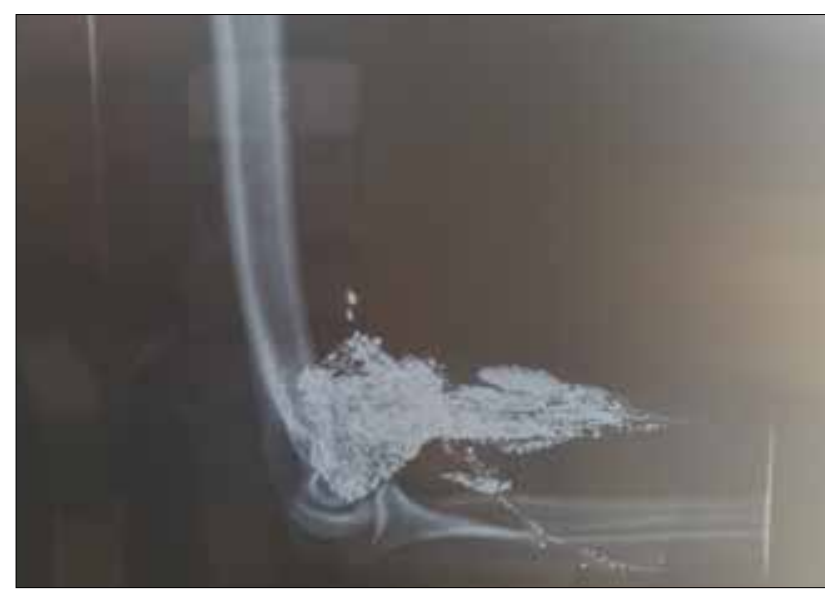

Fig. 2. Radiograph of the right elbow.

soft tissues surrounding the sites of intravenous injection (Figs 1 and 2) as well as numerous small hyperdensities throughout the lung fields (Fig. 3), abdomen and pelvis (Fig. 4)

Blood mercury level is indicative of short-term exposure and its measurement is not of great benefit in chronic exposure; $;^{[1]}$ therefore, this was not tested. Hair mercury levels are a good indication of 


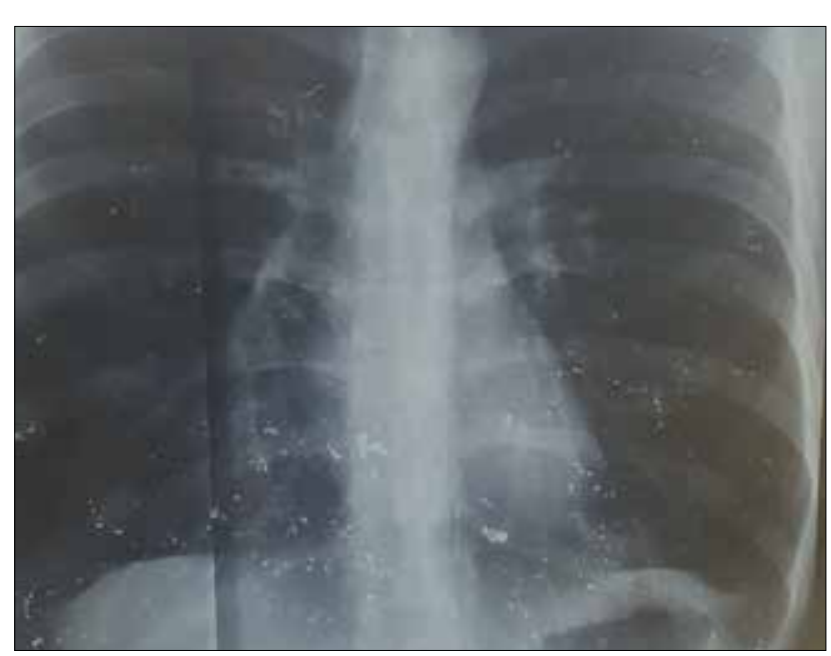

Fig. 3. Radiograph of the chest.

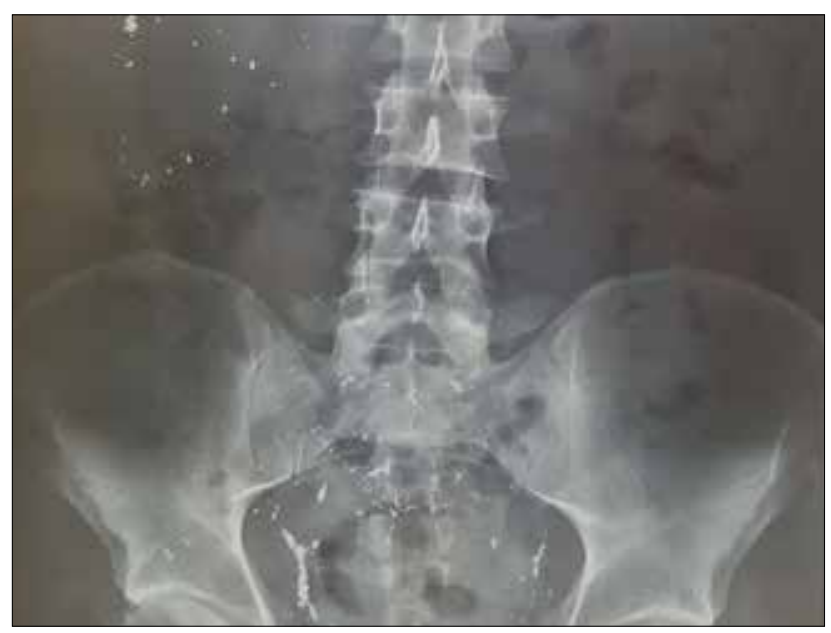

Fig. 4. Radiograph of the abdomen and pelvis.

Table 1. Initial blood analysis results of patient

\begin{tabular}{|c|c|c|}
\hline Component & Value & $\begin{array}{l}\text { Reference } \\
\text { range }^{*}\end{array}$ \\
\hline Urea $(\mathrm{mmol} / \mathrm{L})$ & 11.5 & $2.1-7.1$ \\
\hline Creatinine $(\mu \mathrm{mol} / \mathrm{L})$ & 129 & $64-104$ \\
\hline Total protein $(\mathrm{g} / \mathrm{L})$ & 77 & $60-78$ \\
\hline Albumin $(\mathrm{g} / \mathrm{L})$ & 46 & $35-52$ \\
\hline Alanine transaminase $(\mu / \mathrm{L})$ & 7 & $10-40$ \\
\hline Aspartate transaminase $(\mu / L)$ & 18 & $15-40$ \\
\hline C-reactive protein $(\mathrm{mg} / \mathrm{L})$ & 35 & $<10$ \\
\hline Thyroid-stimulating hormone (mU/L) & 1.29 & $0.27-4.2$ \\
\hline Haemoglobin $(\mathrm{g} / \mathrm{dL})$ & 14.8 & $13.4-17.5$ \\
\hline Urinary mercury $(\mu \mathrm{g} / \mathrm{mL})$ & $>150000$ & $2.5^{\dagger}$ \\
\hline
\end{tabular}

chronic exposure leading to toxicity; ${ }^{[1]}$ however, following discussion with the National Institute for Occupational Health (NIOH), Johannesburg, SA, considering the cost and already significantly raised urinary levels, it was decided not to test hair levels.
The patient was admitted with an acute kidney injury for treatment with intravenous fluids and further investigation of the mercury injection and bipolar mood disorder.

On subsequent psychiatric evaluation of the patient, further information was obtained. The patient's suicide attempt 3 months previously was precipitated by severe stressors at the time, including loss of employment, involvement in a motor vehicle accident with resultant loss of his car, and his father having suffered from a cerebral vascular accident. These events were subsequently followed by mood symptoms, resulting in the mercury injection. The patient was reportedly not experiencing mood or manic symptoms prior to these stressors, according to his mother, despite having defaulted treatment for bipolar mood disorder. He was not experiencing any psychotic or ictal symptoms at the time of admission. He did describe a history of panic attacks since the age of 20 , during which he experiences sudden fear, palpitations, shortness of breath, shaking and lightheadedness, often associated with agoraphobia. Further stressors included an experience of date rape at the age of 22, shortly before the index episode with the first suicide attempt. Treatment with sodium valproate and lamotrigine was started at that stage. On mental state examination, he was noted to have a low mood with no features of major depression, mania or hypomania, and was not suicidal. The patient was restarted on lamotrigine.

Because of the appearance of multiple hyperdensities within the lung field on chest X-ray, the patient was discussed with staff at the respiratory unit at the referral hospital. No further treatment was advised at this point, but discussion with the nephrology department was suggested to assess further treatment options and the possibility of dialysis. Results of blood tests done on admission are shown in Table 1.

During admission, the patient's creatinine level increased to $169 \mu \mathrm{mol} / \mathrm{L}$, urea level was $13 \mathrm{mmol} / \mathrm{L}$ and electrolytes were normal. Urine testing gave a urine protein:creatinine ratio of $0.33 \mathrm{~g} / \mathrm{mmol}$ (nor$\mathrm{mal}<0.015 \mathrm{~g} / \mathrm{mmol}$, nephrotic range $>0.350 \mathrm{~g} / \mathrm{mmol}$; references from the NHLS, Johannesburg). After discussion with the nephrology departments of both Charlotte Maxeke Academic Hospital, Johannesburg, and Groote Schuur Hospital, Cape Town, it was decided that no benefit would be derived from dialysis, but long-term chelation would most likely be necessary.

The case was further referred to the Department of Clinical Pharmacology at the University of Cape Town, which, in discussion with the Medicines Control Council (MCC), advised lifelong treatment with penicillamine.

Documented consent was obtained from the patient for the writing of this case report and the publication of radiographs and blood analysis results.

\section{Discussion}

Organic mercury toxicity often leads to gastrointestinal, neurological and pulmonary symptoms, as organic mercury deposits in the kidneys and liver and in the central nervous system. ${ }^{[2]}$

Most documented clinical sequelae of mercury toxicity are from exposure to vaporised elemental mercury or ingestion of other forms of mercury. ${ }^{[1]}$ Initial signs and symptoms may include cough, pleuritic chest pain, stomatitis, gingivitis, nausea, vomiting and diarrhoea, conjunctivitis and dermatitis.

Prolonged exposure can lead to neurological and psychiatric symptoms, such as insomnia and tremors. A traditional pathognomonic feature of mercury toxicity is known as erethism mercurialis, consisting mainly of neurological manifestations. Features include change in personality, anxiousness, irritability, insomnia, depression and drowsiness. ${ }^{[1]}$ However, the effect on the central nervous system in pre-existing 
mental illness remains to be seen. Rare cases of nephrotic syndrome have been described. Renal tubular dysfunction has also been noted. ${ }^{[1]}$

A diagnosis of mercury toxicity is confirmed in blood and 24-hour urine collection levels, but is dependent on whether the patient has had acute or prolonged exposure.

Treatment strategies include removing the source of exposure if possible, bronchodilators for respiratory complications, and careful fluid and electrolyte management for patients with gastrointestinal features.

Chelation therapy increases urinary excretion of mercury and has been shown to improve clinical manifestations ${ }^{[3]}$ Chelation is recommended by the World Health Organization for severe mercury toxicity.

Toxicity from intravenous injection of mercury usually occurs in relation to attempted suicide, accidental exposure, or possibly through experimentation by drug addicts. ${ }^{[4]}$ Intravenous injection of mercury has been shown to be less detrimental than ingestion or inhalation. The heavy metal moves to dependent areas and extravasates into tissues. It is then transported via the circulation, where it tends to lodge in the lungs and mediastinum and is also known to reach other organs over a period of time. ${ }^{[5]}$

Treatment strategies for intravenous injection are still being explored, but some show areas of promise. Chelation is of benefit; however, the effect of long-term chelation for chronic parenteral exposure is still uncertain. ${ }^{[6]}$ The excision of deposits is also a suggested strategy. ${ }^{[7]}$

Currently, penicillamine is a section 21 substance according to the Medicines and Related Substances Act 101 of 1965, regulated by the MCC. The hospital pharmacy involved in this case is trying to procure the drug.

Other treatment options include dialysis and surgical removal of granulomas and subcutaneous deposits, which have shown benefit. ${ }^{[7]}$
In discussion with the patient it was ultimately decided to treat him medically at this stage and consider surgical intervention later.

Further psychiatric evaluation and monitoring was suggested based on the patient's pre-existing mental state, and the possibility of neuropsychiatric manifestations of mercury toxicity as described above. Suggested further monitoring includes regular renal function testing, and dialysis could be considered depending on the burden of disease. A multidisciplinary approach involving dental and ophthalmology assessments to screen for the possible complications described above, as well as a surgical review to assess the possibility of deposit excision, may be recommended in similar cases.

The patient is waiting for penicillamine delivery via a section 21 application and is attending the outpatient department at Tambo Memorial Hospital, where renal function monitoring continues and psychiatric progression of the disease is being monitored.

. Beauchamp G, Kusin S, Elinder C. Mercury Toxicity. UpToDate. 2016. https://www.uptodate.com/ contents/mercury-toxicity (accessed 23 August 2016).

. Kumar P, Clark M. Clinical Medicine. Edinburgh: Saunders, 2002:984.

3. Zhang J. Clinical observations in ethyl mercury chloride poisoning. Am J Ind Med 1984;5(3):251-258. http://dx.doi.org/10.1002/ajim.4700050308

4. Garg S, Wale J, Yadav P. Elemental mercury poisoning caused by subcutaneous and intravenous injection: An unusual self-injury. Indian J Radiol Imaging 2010;20(2):147. http://dx.doi.org/10.4103/0971-3026.63056 . Gopalakrishna A, Pavan Kumar T. Intravenous injection of elemental mercury: A report of two cases. Indian J Plast Surg 2008;41(2):214. http://dx.doi.org/10.4103/0970-0358.44942

6. McFee R, Caraccio T. Intravenous mercury injection and ingestion: Clinical manifestations and management. J Toxicol Clin Toxicol 2001;39(7):733-738. http://dx.doi.org/10.1081/ctt-100108515

7. Netscher D, Friedland J, Guzewicz R. Mercury poisoning from intravenous injection: Treatment by granuloma resection. Ann Plast Surg 1991;26(6):592-596. http://dx.doi.org/10.1097/00000637-199106000-00018 